\title{
A new MPC scheme for damping wide-area electromechanical oscillations in power systems
}

\author{
D. Wang, M. Glavic, Senior Member, IEEE, and L. Wehenkel
}

\begin{abstract}
This paper introduces a new Model Predictive Control (MPC) scheme to damp wide-area electromechanical oscillations. The proposed MPC controller, based on a linearized discrete-time state space model, calculates the optimal input sequence for local damping controllers over a chosen time horizon by solving a quadratic programming problem. Local controllers considered include: Power Systems Stabilizers (PSSs), Thyristor Controlled Series Compensators (TCSCs) and Static Var Compensators (SVCs). The MPC scheme is realized and tested first in ideal conditions (complete state observability and controllability, neglecting communication and computing delays). Next, the effects of state-estimation errors, computation and communication delays, and of the number and type of available local damping controllers are studied in order to assess the versatility of this scheme. Realistic simulations are carried out using a 16 generators, 70 bus test system.
\end{abstract}

Index Terms-MPC, inter-area oscillations, state-estimation errors, delay, controllability.

\section{INTRODUCTION}

Slow inter-area electromechanical oscillations take on the form of relative rotor motions and sustained power oscillations in transmission lines. Long transmission distances over weak grids, highly variable generation patterns and heavy loading tend to increase the probability of appearance of such oscillations [1], [2]. They can lead to generator outages, line tripping, network splitting and even blackouts [3].

PSSs, TCSCs or SVCs are already used in different countries for damping wide area oscillations. It is however not always easy to tune their control parameters for obtaining satisfactory and robust damping control effects, specially in the context of very large scale systems subject to uncertainty on the location of generation, of load patterns, and network topology.

Wide Area Measurement Systems (WAMSs), together with recent developments in optimal control and computing, could help to design more effective adaptive control schemes for damping inter-area oscillations. For example, defining the differences between post-fault transmission power and its steady state value as a cost function, paper [4] optimizes TCSC parameters based on sensitivity analysis to provide the maximum damping for various operation conditions. Paper [5] introduces angular speeds of remote generators to a PSS to improve its damping to inter-area oscillations.

Based on WAMS, we propose in the present paper a new MPC scheme that can adaptively optimize inputs of

D. Wang, M. Glavic and L. Wehenkel are with the Department of Electrical Engineering and Computer Science, University of Liège, SartTilman, B28 - Liège B4000, Belgium. E-mail: Da.Wang@student.ulg.ac.be, glavic@montefiore.ulg.ac.be, L.Wehenkel@ulg.ac.be. controllers, like PSSs, TCSCs, or SVCs, in order to more effectively damp wide-area oscillations.

MPC has been a very popular method in the process control industry [6]. At a control instant, the MPC algorithm computes an open-loop sequence of inputs in order to optimize future plant behavior. The first input in the optimal sequence is injected into the plant, and the entire optimization is repeated at subsequent control steps [7]. In the field of power systems, there are also some publications that propose to use MPC for emergency alleviation of thermal overloads [8], [9], voltage instability control and stability enhancement [10][13], transient stability and oscillations control [14], [15]. Relevant to the approach of this paper are works presented in [14], [15]. Paper [14] designs a non-linear model predictive controller based on WAMS to enhance transient stability. Defining deviations of the predicted output from reference and control input increments as an objective function, paper [15] proposes a model predictive adaptive control based on a Single Machine Infinite Bus (SMIB) model to damp interarea oscillations in a four-generator system. Compared with the above works, the contributions of the present paper are:

- The proposed MPC scheme can calculate the optimal control inputs for various damping controllers, so as to coordinate these controllers effectively.

- It uses a state-space model of a multi-generator power system and does not use SMIB models [14], [15].

- It can deal with various operation and control constraints which are directly incorporated into the MPC optimization problem.

- It works satisfactorily in presence of realistic stateestimation errors and delays, and is able to accommodate different numbers and types of available local damping controllers.

The rest of this paper is structured as follows. In section II we outline proposed MPC scheme in terms of used model, quadratic optimization problem formulation and state estimation error correction. In the same section the impact of delay in computing and sending control signals to local controllers, is discussed. Test system and simulation parameters are given in section III. Section IV details simulation results obtained in different conditions, while section $\mathrm{V}$ offers some conclusions and future research directions.

\section{OUTLINE OF THE PROPOSED MPC SCHEME}

Fig. 1 illustrates our MPC scheme, which uses a linearized model of the power system's discrete-time dynamics.

We suppose that this model is refreshed from time to time by the Energy Management Systems (EMS) of the control centers, 


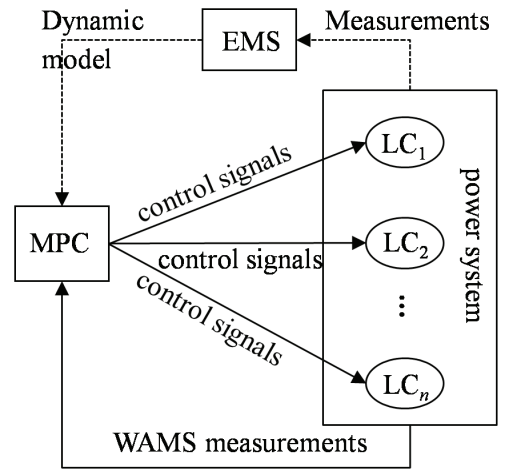

Fig. 1. Schematic diagram of the proposed MPC scheme

following changes of the load level, the generation schedule and the grid topology. In real-time, the MPC controller collects WAMS measurements $\hat{x}(t)$ of the system state ${ }^{1}$ at discrete measurement times (in our simulations, we use $\Delta t=0.1 s$ ). It then uses its model in order to compute an open loop sequence of the control variables $u$ over a certain time horizon composed of $H$ steps of length $\Delta t$, it applies the controls determined for the first period of $\Delta t$ seconds and then waits for the next set of measurements to be received in order to start this computation again (we will use $H=15$, ie. a horizon of $\Delta T=1.5 \mathrm{~s}$ ). We next detail our MPC formulation.

\section{A. Non-linear time domain simulation and discrete time lin- earized dynamic MPC model}

In our simulations, we have replaced the real-system by a non-linear time domain simulation software from the MATLAB Power System Toolbox (PST) [3]. We also used the svm-mgen module for small signal stability analysis of PST to derive the linearized continuous time model

$$
\left\{\begin{array}{l}
\dot{x}=A_{c} x+B_{c} u \\
y=C_{c} x
\end{array}\right.
$$

where, $x \in R^{m_{x}}$ is a vector of state variables, $u \in R^{m_{u}}$ is a vector of inputs, $y \in R^{m_{y}}$ is a vector of outputs. Next, we inferred from Eqn (1) the transition for a small step of $\delta$ seconds (we use $\delta=0.005 \mathrm{~s}$ in our simulations) by:

$$
\left\{\begin{array}{l}
x(t+\delta)=\left(\delta A_{c}+I\right) x(t)+\delta B u(t) \\
y(t)=C x(t)
\end{array}\right.
$$

yielding a discrete-time dynamics (for time steps $k \delta$ ) given by

$$
\begin{aligned}
& x[k+1]=A x[k]+B u[k] ; y[k]=C x[k], \\
& \text { with: } A=\left(\delta A_{c}+I\right) ; B=\delta B_{c} ; C=C_{c} .
\end{aligned}
$$

\section{B. Quadratic optimal control problem formulation}

At time $t$, based on the estimation $\hat{x}(t)$ of the current system state (obtained from a state estimator), the predicted outputs $\hat{y}(t+k \delta)=\hat{y}[k]$ over the next horizon are obtained by iterating

\footnotetext{
${ }^{1}$ To get the actual system state from WAMS measurements, one normally would need a state estimator, but this is out of the scope of the present paper.
}

Eqn (2) $K$ times by using $\hat{x}(t)$ as initial state (we use $K=300$ in our simulations, corresponding to $K \delta=\Delta T=1.5 \mathrm{~s}$ ):

$$
\left[\begin{array}{c}
\hat{y}[1] \\
\hat{y}[2] \\
\vdots \\
\hat{y}[K]
\end{array}\right]=P_{x} \hat{x}(t)+P_{u}\left[\begin{array}{c}
u[0] \\
u[1] \\
\vdots \\
u[K-1]
\end{array}\right]
$$

where $P_{x}$ and $P_{u}$ are given by

$$
P_{x}=\left[\begin{array}{c}
C A \\
C A^{2} \\
\vdots \\
C A^{K}
\end{array}\right] \quad P_{u}=\left[\begin{array}{cccc}
C B & 0 & \ldots & 0 \\
C A B & C B & \ldots & 0 \\
\vdots & \vdots & \ddots & \vdots \\
C A^{K-1} B & C A^{K-2} B & \ldots & C B
\end{array}\right]
$$

Using these equations, the MPC controller solves the following quadratic optimization problem:

$$
\min _{u[\cdot]}\left\{\sum_{i=1}^{K}\left(\hat{y}[i]-y_{r}\right)^{T} Q\left(\hat{y}[i]-y_{r}\right)\right\}
$$

subject to linear inequality constraints $(\forall i=0, \ldots, K-1)$ :

$$
\begin{aligned}
& u_{\min } \leq u[i] \leq u_{\max } \\
& z_{\min } \leq z[i+1] \leq z_{\max }
\end{aligned}
$$

where $Q$ is a symmetric positive definite matrix of weight factors, $y_{r}$ is the vector of output targets, and $z$ is a vector of constrained operation variables like currents or voltages.

In our simulations we used Quadprog, a MATLAB function, in order to compute the solution of this quadratic programming problem. The first part (i.e. $u[0] \ldots u[\Delta t / \delta-1]$ ) of this solution is injected into the local controllers (LCs, in Fig. 1), and the calculation is repeated at the next measurement step $t+\Delta t$ by using the measured state $\hat{x}(t+\Delta t)$ as input.

\section{Modeling of state-estimation errors}

At any refreshment time $t$, the MPC controller uses the results $\hat{x}(t)$ from a state estimator to compute the optimal inputs for LCs. Consequently, the state-estimation imprecision may have a detrimental effect on the MPC controller's decisions.

In order to compensate for this imprecision, we assume that at each refreshment time $t$ we dispose also of an accurate measurement of the actual outputs $y(t)$ in addition to the estimated state $\hat{x}(t)$. This allows us to compute the difference $d(t)=y(t)-C \hat{x}(t)$ between the actual outputs and their estimated value, and hence to correct Eqn (4) as in [6], [7], namely:

$$
\left[\begin{array}{c}
\hat{y}[1] \\
\hat{y}[2] \\
\vdots \\
\hat{y}[K]
\end{array}\right]=P_{x} \hat{x}(t)+P_{u}\left[\begin{array}{c}
u[0] \\
u[1] \\
\vdots \\
u[K-1]
\end{array}\right]+\left[\begin{array}{c}
I \\
I \\
\vdots \\
I
\end{array}\right] d(t)
$$

where

$$
d(t)=y(t)-C \hat{x}(t)
$$

It is thus assumed that $d(t)$ is refreshed each time a new state estimate is collected, and then remains unchanged over the entire prediction horizon used to compute the controls. 


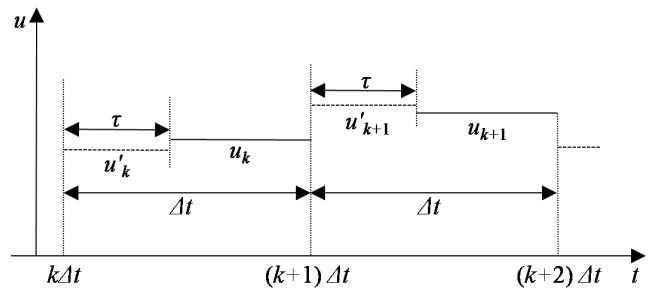

Fig. 2. inputs $u$ and delay $\tau$

\section{Consideration of time delays}

Various delays, in measurements acquisition, computation and sending inputs to local controllers, could be involved in the implementation of the proposed MPC scheme [7]. It is therefore important to asses the impact of such delays on the performance of the proposed control scheme. We study the impact of delays in computation and sending inputs to local controllers by still assuming that all measurements are taken synchronously, and then exploited with a common delay. This is illustrated in Fig. 2 assuming a common delay of $\tau$. We assume that this delay is smaller than the refreshment period $\Delta t$ of the MPC scheme.

To take the delays into account in a proper way, we proceed as follows. At every control refreshment instant $t=k \Delta t$, the MPC controller computes inputs for the next period until $(k+1) \Delta t$ and also for the subsequent period until $(k+2) \Delta t$. Because of the delay $\tau$, inputs calculated at $t=k \Delta t$ are only applied from $k \Delta t+\tau$. Thus, between $k \Delta t$ and $k \Delta t+\tau$, inputs calculated at the previous refreshment time are applied, while those computed from the measurements at time $k \Delta t$ are applied during the period $k \Delta t+\tau$ until $(k+1) \Delta t+\tau$.

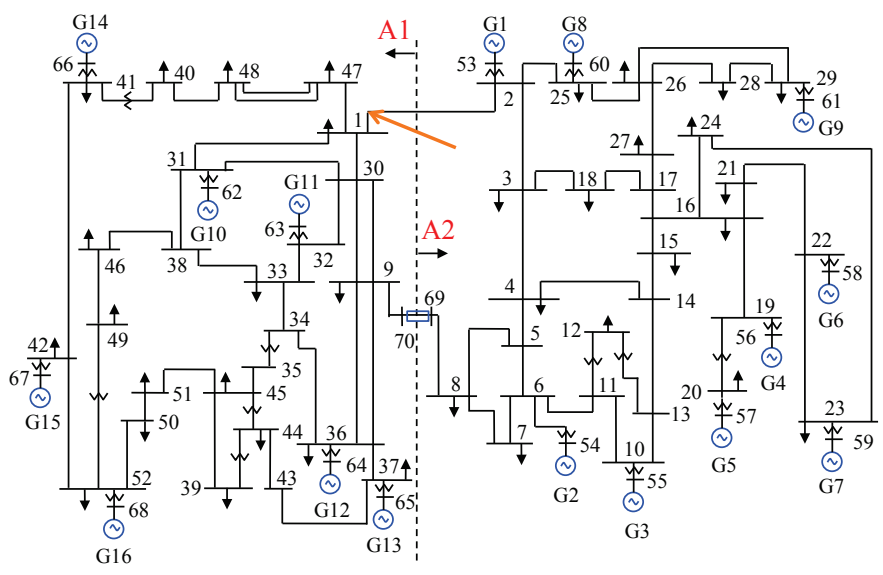

Fig. 3. 16 generators / 70 bus system

\section{TEST SYSTEM AND SIMULATION PARAMETERS}

In order to illustrate the proposed control scheme, we use the system of Figure 3. A TCSC is installed between buses 69 and 70, and there is a PSS on each generator. We use the dynamic models of Ref. [3] to simulate the system response and to derive the linearized models used by MPC. The system contains two areas: A1 and A2. In our test scenarios, a
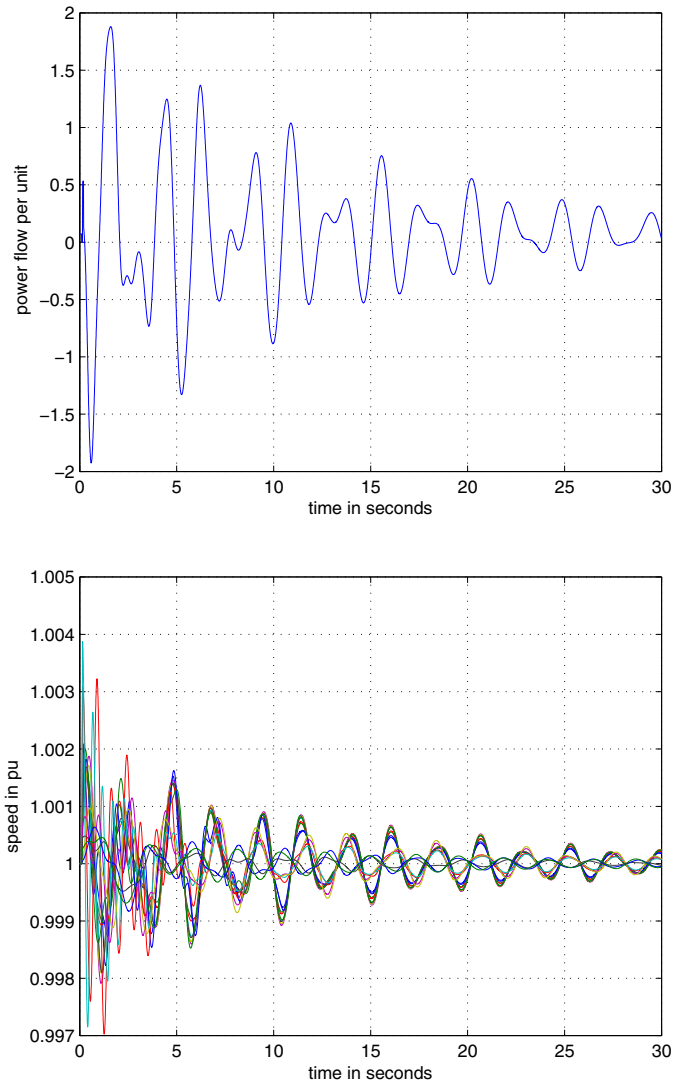

Fig. 4. Response without MPC controller: (top) active power of tie-line 1-2; (bottom) angular speeds

temporary three-phase short-circuit to ground at bus 1 causes oscillations between A1 and A2; the fault is cleared by opening successively the breakers at the near and far end of the line and then closing them after a short delay. Figure 4 shows respectively the temporal evolution over a period of 30s of the power flow through line 1-2 and the angular speeds of all generators, when no MPC controller is used. We observe sustained, slowly damped oscillations.

We next introduce the MPC controller to coordinate PSSs and TCSC. In this case, the MPC state vector $x$ contains $6 \times 16$ generator states, $3 \times 16$ exciter states, $3 \times 16$ PSS states, $3 \times 16$ turbine governor states and 1 TCSC state. Output $y$ is the vector of angular speeds, which reference $y_{r}$ is the unit vector of dimension 16. Input vector $u$ consists of 17 supplementary inputs: 16 of them for PSSs and one for TCSC, which is subject to $-0.1 \leq u \leq 0.1$. Because of limitations in the PST model implementations, we used equivalent supplementary inputs to exciters to substitute for those supplementary inputs to PSSs because the PSS damps oscillations by adding a voltage component to the exciter.

As previously mentioned, we used in our simulations a control horizon of $H=15$ discrete steps of $\Delta t=0.1$ s (i.e. $1.5 \mathrm{~s}$ long) while we used a time step $\delta=0.005$ for formulating the MPC dynamics and optimization problem. To assess the dynamics of the power system we simulate and observe its response in various conditions over a period of $20 \mathrm{~s}$ or $30 \mathrm{~s}$. 

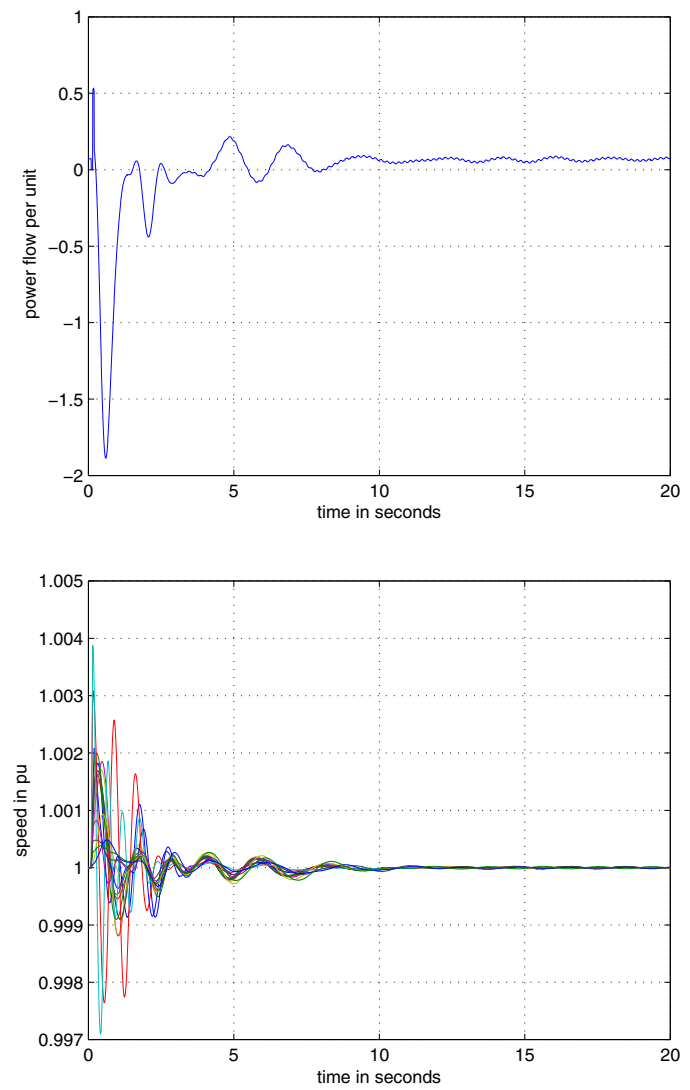

Fig. 5. Response using PSSs and TCSC in ideal conditions: (top) active power of tie-line 1-2; (bottom) angular speeds

In the objective function (5) all controls are weighted uniformly and independently, i.e. $Q$ is the $16 \times 16$ identity matrix. In order to reduce computing times for solving the MPC optimization problem, we further imposed that the values of $u$ over the horizon of 1.5 seconds may take only three different values, namely $u[k]=\alpha$ for the first 20 steps $(0.1$ seconds), $u[k]=\beta$ for the next 20 steps $(0.1$ seconds $)$, and $u[k]=\gamma$ for the remaining 260 steps (1.3 seconds). This reduces significantly the computational burden ${ }^{2}$ without jeopardizing the performances.

\section{Simulation RESUlts}

In this section we present detailed results starting with ideal conditions where all PSSs and a TCSC are considered as available local controllers. Next, using the same available LCs, the performances of proposed MPC scheme are assessed in presence of state estimation errors. Further results using different numbers and types of available controls are also included.

\section{A. Results in ideal conditions}

Ideal conditions assume complete state observability and controllability, with telecommunication and computation delays neglected.

\footnotetext{
${ }^{2}$ by reducing the number of optimization variables from $17 \times 300=51,000$ to $17 \times 3=51$
}
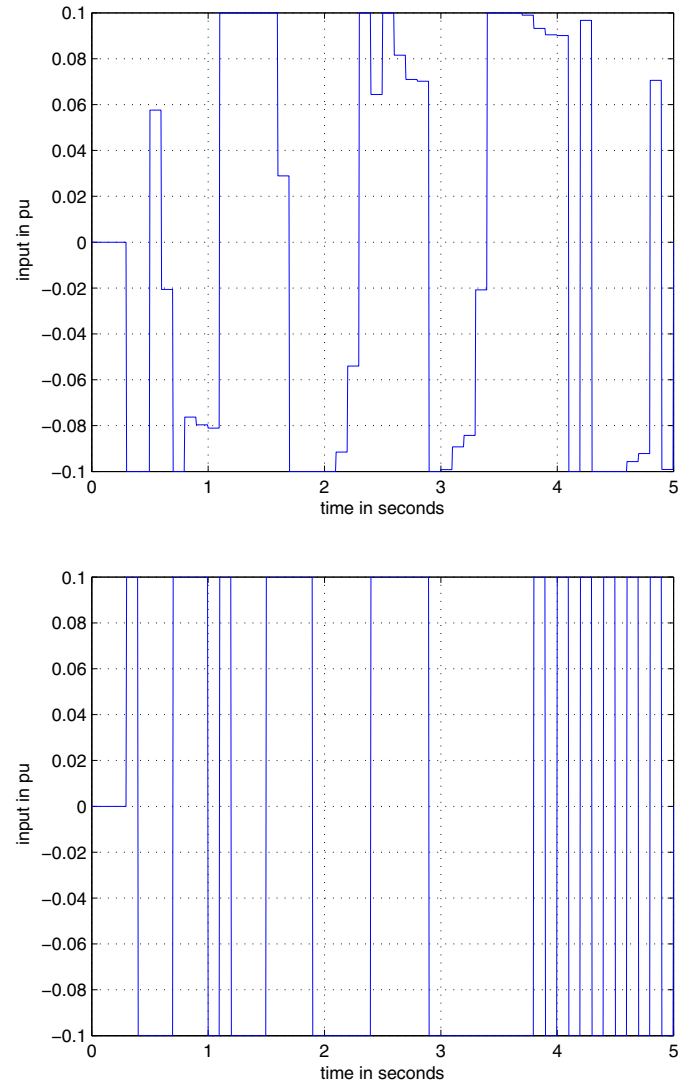

Fig. 6. MPC control signals: (top) PSS signal of generator 1; (bottom) TCSC signal

Controlled system response over time a period of $30 \mathrm{~s}$ of the power flow through line 1-2 and the angular speeds of all generators is given in Figure 5. Compared with the system response without MPC (Figure 4), we clearly observe that the proposed MPC scheme damps the power oscillations of the tie-line and angular speeds of all generators, more efficiently (settling time is decreased to approximately 10 seconds). Figure 6 shows the control signals computed by the MPC scheme for a representative PSS and the TCSC over the first period of $5 s$.

\section{B. Results with state-estimation errors}

In order to mimic state estimation errors, we consider additive random noise. Specifically, we use uniformly distributed pseudorandom errors of $\pm 10 \%$ that are superimposed on the exact states so as to represent state-estimation errors.

The results are shown in Figure 7 without (blue curves) and with (red curves) state estimation errors correction.

As expected, inaccuracies introduced by state estimation errors affect the performances of the MPC scheme in terms of both magnitude of oscillations and settling time. On the other hand, we observe that the correction of state estimation errors according to eqn. (7) considerably improves the performance of the control scheme (see red curves in Figure 7), both in terms of angular speeds and in terms of damping the power flow oscillations in line 1-2. 

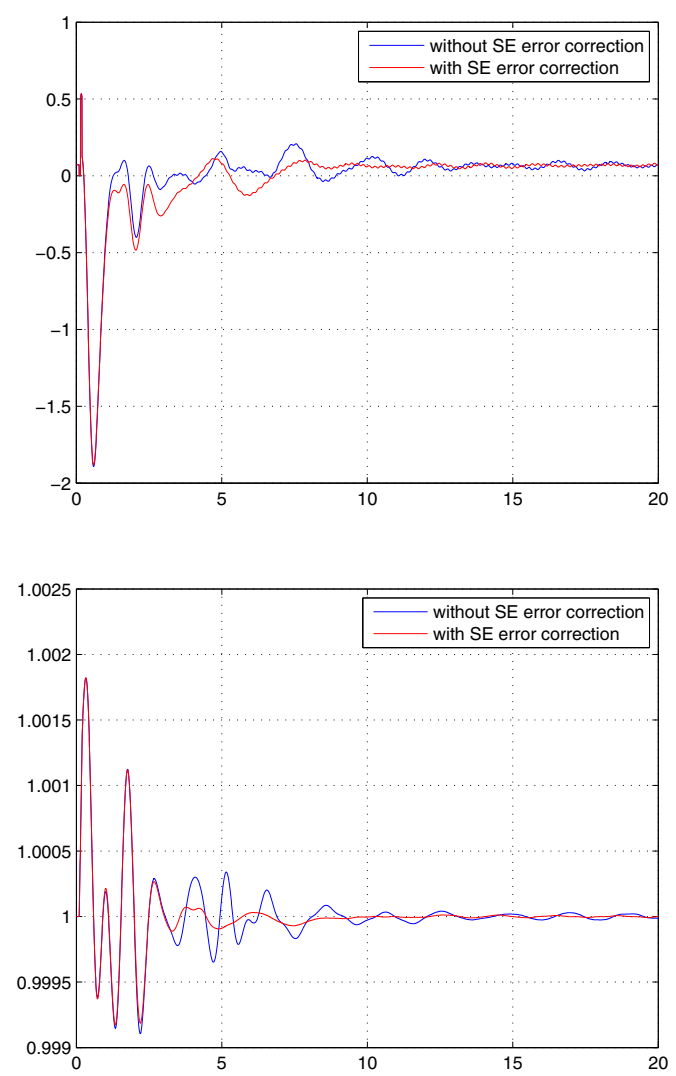

Fig. 7. Response using PSS and TCSC with state-estimation errors: (top) active power of tie-line 1-2; (bottom) angular speed of generator 1

C. Taking into account delays in computing and sending control signals

Time delays in computing and sending controls signals to LCs may vary in practice. There are two possibilities, as discussed in [7]: to apply control signals as soon as they are available or the signals are applied after a specific time interval. As explained before, we consider later option.

We suppose that a delay of $\tau=0.05 \mathrm{~s}$ in computing and sending control signals to all available LCs (the same as in previous section) is introduced. System response under this assumption is shown in Figure 8.

We observe, indeed, that the control effects become worse because the delay weakens MPC effectiveness. But compared with Figure 4, the system performance with the MPC controller is still superior to that without MPC.

\section{Effect of unavailabilities of some local controllers}

So far, we assumed that all 16 PSSs and the TCSC are available to the MPC controller and are able to receive supplementary control inputs. In this subsection we consider the case where several PSSs and the TCSC are not available to the MPC controller.

In our simulations, we assumed that the MPC controller is aware of the remaining available LCs and hence adjusts its strategy by computing control inputs only for these latter LCs (non-available LCs are hence removed from the optimal
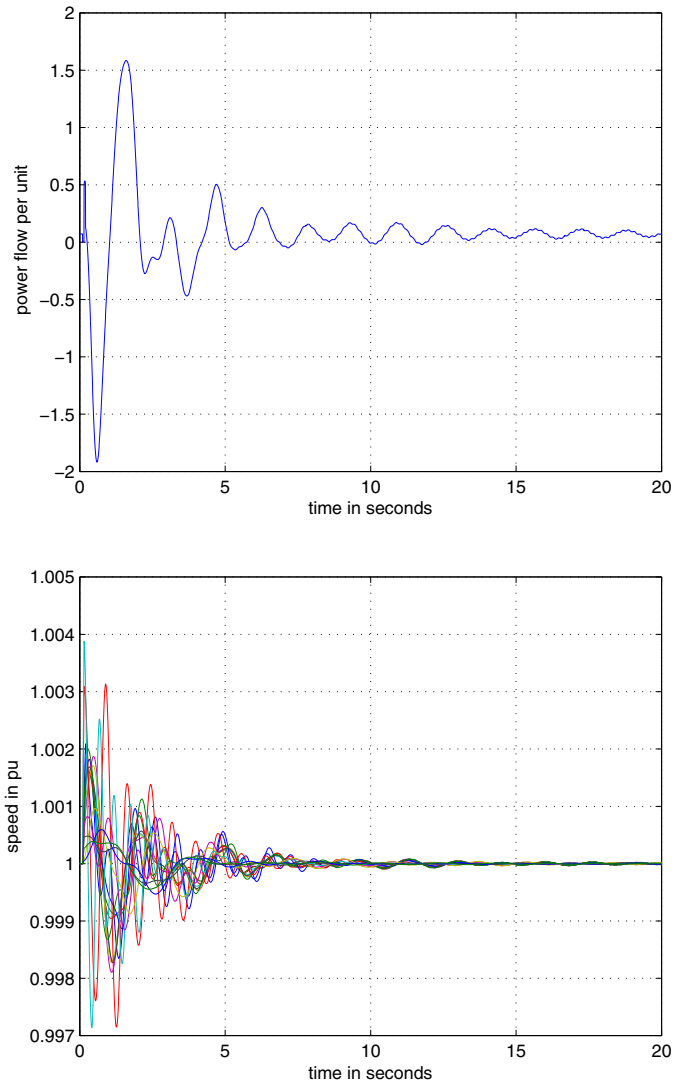

Fig. 8. Response using PSS and TCSC with with a 0.05 s delay: (top) active power of tie-line 1-2; (bottom) angular speeds

control problem formulation, but the effect of the local control loops are still modeled in the system dynamics).

In other words, we do not assess the robustness of MPC with respect to unnoticed controller failure but rather study the effect of decreased system controllability. We believe that this investigation well illustrates fault-tolerance capability of the proposed MPC in the practical case of identified and isolated LC failure (generally, fault detection and isolation for this type of failure is not considered to be a difficult problem [7]).

The blue curves of Figure 9 show the system response when six PSSs (PSS1-3, PSS10-12) and TCSC are not included in MPC control scheme. Performances become slightly worse. When eight PSSs (PSS1-4, PSS10-13) and TCSC are not included in the MPC scheme (this represents $53 \%$ of all available damping controllers) performances further deteriorate, but they remain still acceptable compared to the uncontrolled case, as shown by the red curves of Figure 9.

In the literature, it has been noticed that MPC schemes have certain inherent fault-tolerance properties [7]. We plan to investigate this property in our future work so as to further assess the robustness of the proposed MPC scheme for interarea oscillation damping.

\section{E. Incorporating additional control devices}

One of the key advantages in using MPC to damp electromechanical oscillations is its flexibility to cope with any 

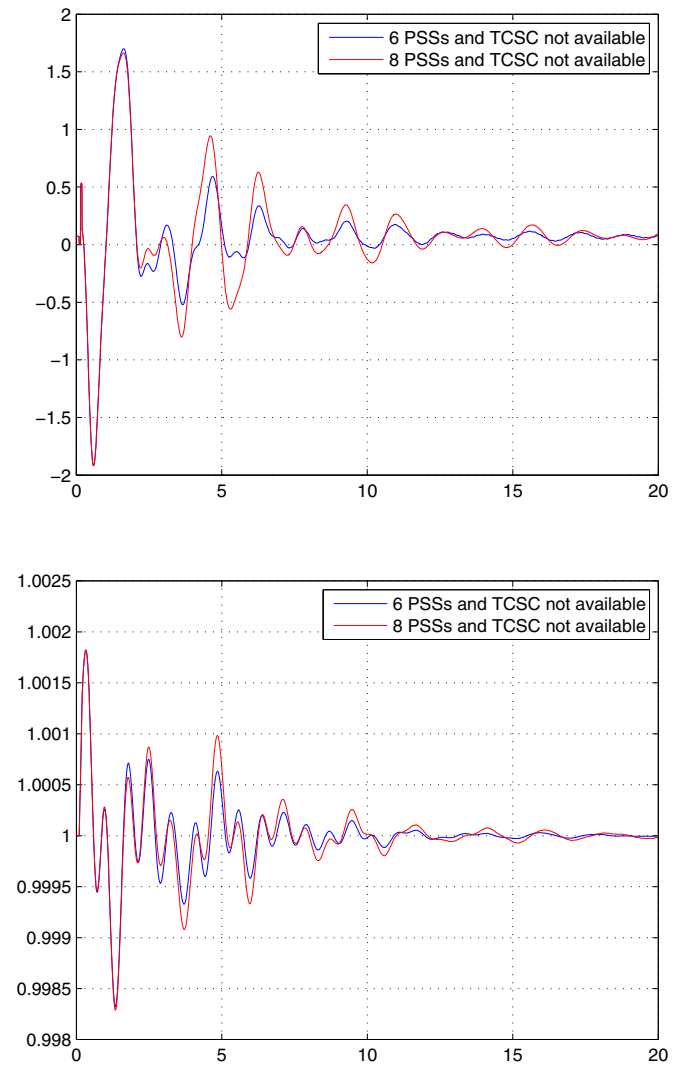

Fig. 9. Reduced number of available local controllers: (top) active power of tie-line 1-2; (bottom) angular speed of generator 1

kind of control device. To illustrate this in the context of our test system, we consider in this subsection the possibility to use another type of local controller, namely a Static Var Compensator (SVC). Thus we introduce a new type of LC (an $\mathrm{SVC}$ ) in addition to the already considered ones. This SVC is installed in bus1.

Figure 10 displays the for this case (we assume availability of all PSSs and the TCSC) while Figure 11 shows the control inputs for PSS1, TCSC and SVC.

These results clearly show that the proposed MPC is indeed able to effectively incorporate a new control device and successfully coordinate its control efforts with the other available controls regardless of their type. We leave for further research the question of what would be the best mix of LCs in any given practical context.

\section{CONCLUSION}

In order to help in the design of wide-area control schemes for damping electromechanical inter-area oscillations in large scale power systems, we have proposed and investigated in this paper a new Model Predictive Control scheme. This approach is justified by the fact that in modern power systems we can dispose at the same time of good dynamic models, of accurate and fast measurement systems, and of state-of-the-art optimal control formulations and scalable algorithms to solve them in real-time with available computing platforms.
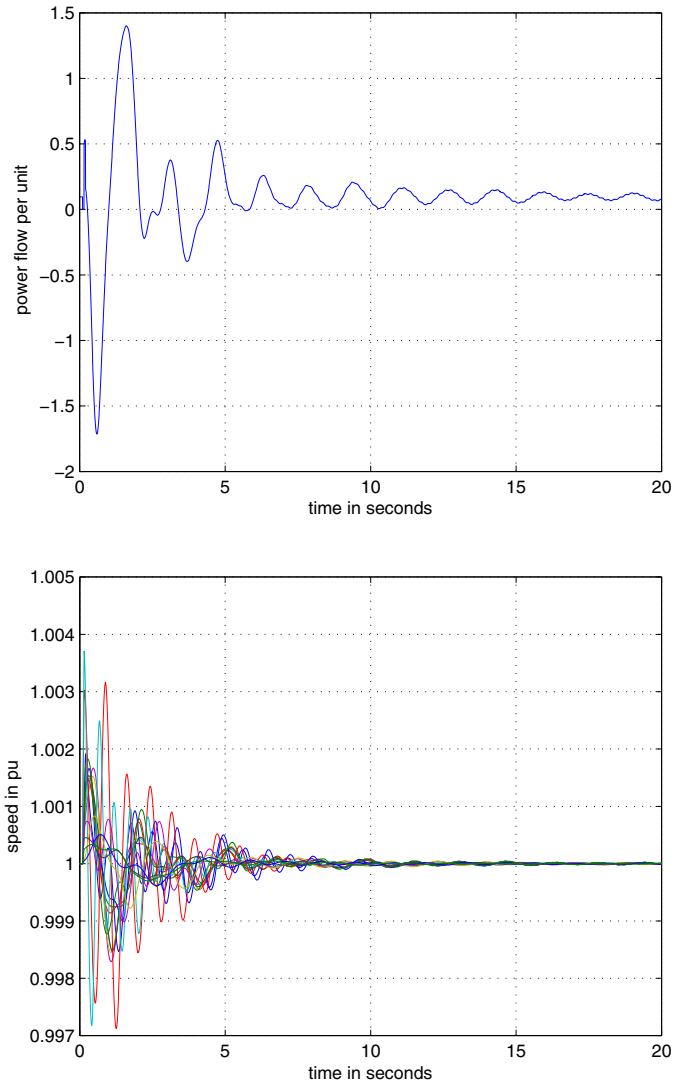

Fig. 10. Response using PSS, TCSC and SVC: (top) active power of tie-line 1-2; (bottom) angular speeds

The proposed MPC scheme calculates all control signals in a centralized way. Encouraging results obtained using a medium sized power system show that this scheme can indeed effectively and quickly damp inter-area oscillations. We also provided additional experiments showing that such MPC schemes can tolerate realistic state-estimation errors and communication and computation delays.

Furthermore, we have illustrated the versatility of the MPC control approach by showing how it can adapt effectively to handle a reduced number of control devices and how it can take advantage of additional control devices.

Overall, the simulation results provided in this paper show that a proper MPC scheme may be quite effective in coordinating a broad diversity of control devices, such as PSSs, TCSCs, and an SVCs, for the damping of inter-area electromechanical power system oscillations.

In our future work, we plan to extend the proposed framework in order to comply with practical constraints of very large scale interconnected power systems. Specifically, we will aim at designing a multi-layer and distributed MPC scheme that we believe is more appropriate for large scale multi-area systems. In such a scheme fast updates of controls would be carried out in the lower layers based on detailed models of smaller sub-areas, while slower updates of controls would be used to coordinate these at the higher layers based on the aggregation of the models of the lower layers. 

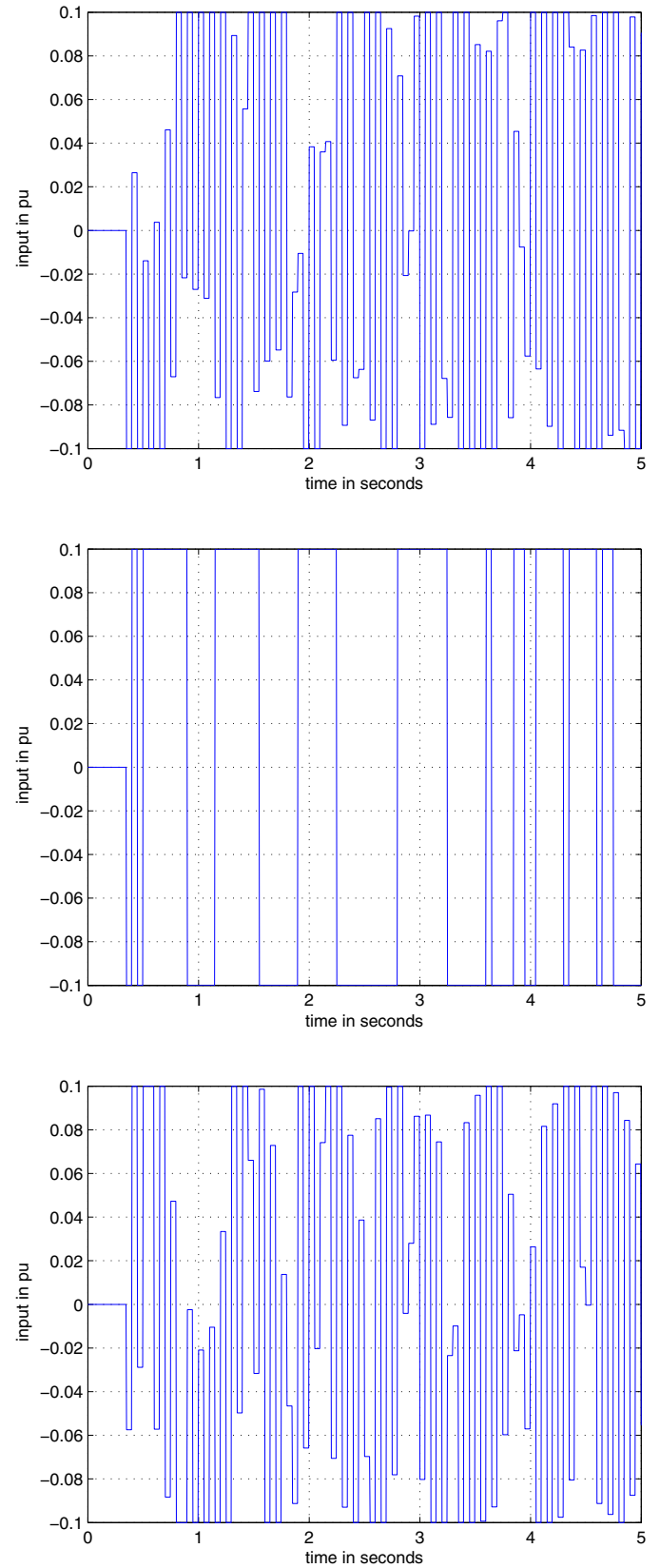

Fig. 11. MPC control signals: (top) PSS signal of generator 1; (middle) TCSC signal; (bottom) SVC signal

\section{ACKNOWLEDGEMENTS}

This paper presents research results of the Belgian Network DYSCO (Dynamical systems, control and optimization), funded by the Interuniversity Attraction Poles Programme initiated by the Belgian State Science Policy Office, and of the PASCAL2 Network of Excellence funded by the European Commission FP7 Programme. The scientific responsibility rests with the authors.

\section{REFERENCES}

[1] J. Lehner, T. Weissbach, G. Scheffknecht, "Oscillation behaviour of the enlarged UCTE power system including the Turkish power system". Proceedings of the 17th IFAC World Congress, Korea, July 6-11, pp. 9404-9409, 2008.

[2] E. Grebe, J. Kabouris, S. Lopez Barba, W. Sattinger, W. Winter, "Low Frequency in the Interconnected System of Continental Europe". Proceedings of IEEE General Meeting 2010, Minneapolis, MN, July 2010.

[3] G. Rogers, Power System Oscillations. Norwell, Massachusets, USA: Kluwer Academic Publishers, 2000.

[4] P. S. Dolan, J. S. Smith and W. A. Mittelstadt, "A study of TCSC optimal damping control parameters for different operating conditions", IEEE Transactions on Power Systems, vol. 10, no. 4, November, pp. $1972-$ 1978, 1995.

[5] C. Li, X. Chen and Z. Ma, "Design of a wide-area PSS for damping inter-area low-frequency oscillations", Proc. of the 5th IEEE Conference on Industrial Electronics and Applications, Georgia, USA, pp. 808-813, 2010.

[6] S. J. Qin, T. A. Badgwell, "A survey of industrial model predictive control technology", Control Engineering Practice, Vol.11, pp. 733764, 2003.

[7] J. M. Maciejowski, Predictive Control with Constraints. Harlow, England: Prentice Hall, 2002.

[8] E. Camponogara, D. Jia, B. H. Krogh and S. Talukdar, "Distributed model predictive control", IEEE Control Systems Magazine, November, pp. 44-52, 2002.

[9] B. Otomega, M. Glavic, and T. Van Cutsem, "Emergency alleviation of thermal overloads using model predictive control", Proc. 2007 IEEE Laussane PowerTech, July, pp. 201-206, 2007.

[10] M. Larsson, D. Kalsson, "Coordinated system protection scheme against voltage collapse using heuristic search and predictive control", IEEE Transactions on Power Systems, vol. 18, no. 3, pp. 1115-1122, Aug. 2003.

[11] I. A. Hiskens, B. Gong, "Voltage stability enhancement via model predictive control of load", Proceedings of Bulk Power System Dynamics and Control VI, pp. 117-124, Cortina dAmpezo, Italy, 2004.

[12] J. Y. Wen, Q. H. Wu, D. R. Turner, S. J. Cheng and J. Fitch, "Optimal coordinated voltage control for power system voltage stability", IEEE Transactions on Power Systems, vol. 19, no. 2, May, pp. 1115-1122, 2004.

[13] J. Lin, R. Kumar, N. Elia, "Model Predictive Control-Based Real-Time Power System Protection Schemes", IEEE Transactions on Power Systems, vol. 25, no. 2, pp. 988-998, May 2010.

[14] S. R. Wagh, A. K. Kamath and N. M. Singh, "Non-linear model predictive control for improving transient stability of power systems using TCSC controllers", Proc. of the 7th Asian Control Conference, Hong Kong, China, pp. 1627-1632, 2009

[15] L. Wang, H. Cheung, A. Hamlyn and R. Cheung, "Model predictive adaptive control of inter-area oscillations in multi-generator power system", Proc. of the IEEE PES General Meeting, Calgary, Canada, pp. 1-7, 2009 . 